\title{
A Converse to Banach's Contraction Theorem
}

\author{
Philip R. Meyers \\ Institute for Basic Standards, National Bureau of Standards, Washington, D. C. 20234
}

(March 3, 1967)

\begin{abstract}
The class of all continuous self-mappings of a metrizable space which can become contractions (in the sense of Banach) under metrics compatible with the topology on the space is characterized. The characterization amounts to a converse to the Contraction Mapping Principle.
\end{abstract}

Key Words: Contractions, functional analysis, metric spaces, topology.

\section{Introduction}

Throughout this paper, $X$ denotes a metrizable topological space and $f: X \rightarrow X$ denotes a continuous map. If $\rho$ is a metric on $X$, then $f$ is called a $\rho$-contraction in case there exists $\lambda \epsilon(0,1)$ which is a contraction constant for $f$ on $(X, \rho)$, i.e., for all $x, y \in X$,

$$
\rho(f(x), f(y)) \leqslant \lambda \rho(x, y) .
$$

Consider the hypothesis $(\mathrm{H})$ that $X$ admits a metric $\rho$ (yielding the correct topology) such that $f$ is a $\rho$-contraction. In a previous paper [1] ${ }^{1}$ we gave conditions on $X$ such that $f$, if for some metric it satisfied (1.1) locally at all points of $X$, would in fact obey $(\mathrm{H})$. Note that such results have a hypothesis which is metric in nature. The present paper is concerned with topological conditions for $(\mathrm{H})$, conditions whose statements do not refer to any particular metric or metrics on $X$.

In view of the Banach Contraction Theorem [2], two conditions which naturally suggest themselves are that, for some $\xi \in X$,

$$
f(\xi)=\xi,
$$

$$
f n(x) \rightarrow \xi \text { as } n \rightarrow \infty \text {, for all } x \in X
$$

These are among the usually stated conclusions of the Contraction Theorem, and so are surely necessary if $(\mathrm{H})$ is to hold for some complete ${ }^{2}$ metric $\rho$. We shall add to them a third condition, an easy (though usually unstated) conclusion of the Contraction Theorem, and then show that these three conditions together are sufficient (as well as necessary) to guarantee $(\mathrm{H})$. The third condition is that there exist an open neighborhood $U$ of $\xi$ such that

$$
\text { (iii) }
$$

$$
f^{\prime \prime}(U) \rightarrow\{\xi\} .
$$

'Figures in brackets indicate the literature references at the end of this paper.

2 Completeness is rather inessential if we are willing to operate with an equivalence class of Cauchy sequences rather than an actual point.
This means that for any open neighborhood $V$ of $\xi$, there is an $n(V)>0$ such that $f^{\prime \prime}(U) \subset V$ for all $n \geqslant n(V)$.

If $(\mathrm{H})$ holds, take $U=\{x: \rho(x, \xi)<1\}$. Since $\lambda<1$ in (1.1), we can for any neighborhood $V$ of $\xi$ choose $n(V)$ so large that for all $n \geqslant n(V)$,

$$
\left\{x: \rho(x, \xi)<\lambda^{\prime \prime}\right\} \subset V,
$$

which implies by (1.1) that $f^{\prime \prime}(U) \subset V$. Thus (iii) is indeed a consequence of $(\mathrm{H})$. It remains to show how (H) can be deduced from (i), (ii), and (iii).

\section{Results and Corollaries}

At this point we state all of the results of the paper. As above, $f$ is a continuous self-mapping of the metrizable topological space $X$.

THEOREM 1: If $\mathrm{f}$ satisfies conditions (i), (ii), and (iii) above, then for each $\lambda \epsilon(0,1)$ there exists a metric $\rho_{\lambda}$ on $\mathrm{X}$, complete if $\mathrm{X}$ admits a complete metric, such that $\mathrm{f}$ is a $\rho_{\lambda}$-contraction with contraction constant $\lambda$.

CoRoLLARY 1.1: If $\xi$ has a compact neighborhood, then (i) and (ii) are sufficient conditions in Theorem 1 .

Corollary $1.2:^{3}$ If $\mathrm{X}$ is compact, and if $\{\xi\}$ is the only nonempty set $\mathrm{D}$ such that

$$
f(D)=D
$$

then the conclusions of Theorem 1 are valid.

THEOREM 2: If some iterate $\mathrm{f}^{\mathrm{m}}$ of $\mathrm{f}$ satisfies conditions (i), (ii), and (iii) then there is a metric $\rho$, complete if $\mathrm{X}$ admits a complete metric, such that $\mathrm{f}$ and all its iterates are simultaneously $\rho$-contractions.

Theorem 2 suggests some further questions. Under what conditions do two mappings which satisfy conditions (i), (ii), and (iii) become contractions under

\footnotetext{
3 This corollary is a restatement of a theorem due to Janos [3]. The proof of Theorem ] uses ideas first used by Janos in his proof of this theorem; the proof has just been published.
} 
the same metric? More generally, when can $X$ be metrized so that a given family of self-mappings simultaneously become contractions? These and similar questions will be addressed in another paper under preparation.

The proof of Theorem 1 involves a somewhat lengthy construction of the desired metric $\rho_{\lambda}$. While this construction will be deferred to the next section, we give here the derivations of the remaining results from Theorem 1 .

To derive Corollary 1.1, it suffices to show that (iii) follows from (i) and (ii) if $\xi$ has a compact neighborhood $C$. Let $U=\operatorname{Int}(\mathrm{C})$, an open neighborhood of $\xi$. Consider any open neighborhood $V$ of $\xi$. For each $x \in C$, there exists by (ii) a smallest $n(x)$ such that $f^{\prime \prime}(x) \epsilon V$ for all $n \geqslant n(x)$. We need only show that

$$
n(V)=\sup \{n(x): x \in C\}
$$

is finite. If not, then $C$ contains a sequence $\left\{x_{i}\right\}$ such that $n\left(x_{i}\right)>i$, and since $C$ is compact we may assume $x_{i} \rightarrow y$ for some $y \in C$. The desired contradiction follows by observing that $n(y)<\infty$, and that by continuity $n(x) \leqslant n(y)+1$ for all $x$ in some neighborhood of $y$.

To derive Corollary 1.2, we show that (ii) follows from the stated hypotheses when $X$ is compact and then use Corollary l.l. Define successively

$$
A_{0}=X, A_{1}=f(X), A_{2}=f^{2}(X)=f\left(A_{1}\right), \text { etc. }
$$

Since $f(X) \subset X$ and if $X \neq\{\xi\}$ the inclusion is proper, the sets $A_{m}$ are a descending sequence of compact nonempty sets. Then $\stackrel{x}{\cap} A_{m}=D \neq \phi$. We show that

$$
f(D)=D
$$

and conclude that $D=\{\xi\}$. It is then a standard argument that diam $\left(A_{m}\right) \rightarrow 0$ and hence $f^{m}(x) \rightarrow \xi$ for $x \in X$. It is clear that

$$
\bigcap_{0}^{\infty} A_{m}=D \supset f(D)=\bigcap_{1}^{\infty} A_{m}
$$

In the other direction choose $x \in D$ and consider the sequence of nonempty compact sets

$$
S_{m}=f^{-1}(x) \cap A_{m} .
$$

Since $\cap S_{m}=f^{-1}(x) \cap D \neq \phi$ 'we have $x \in f(D)$ and $f(D) \supset D$ which completes the proof.

To derive Theorem 2, we show that $f$ obeys conditions (i), (ii), and (iii) for the same point $\xi$ as does its iterate $f^{m}$. Then by Theorem 1 there is a metric $\rho$ and a $\lambda \epsilon(0,1)$ such that $f$ is a $\rho$-contraction with contraction constant $\lambda$. It follows that any iterate $f^{n}$ is a $\rho$-contraction with contraction constant $\lambda<1$.

Since $f^{\prime \prime}(\xi)=\xi$, we have

$$
f^{n m}(f(\xi))=f\left(f^{n m}(\xi)\right)=f(\xi)
$$

for all $n \geqslant 0$. But by (ii) applied to $f^{\prime \prime \prime}$, with $x=f(\xi)$,

$$
\left(f^{m}\right)^{n}(f(\xi))=f^{n m}(f(\xi)) \rightarrow \xi \quad \text { as } n \rightarrow \infty
$$

and so $f(\xi)=\xi$, condition (i) for $f$. For $0 \leqslant k<m$ we have, for any $x \in X$,

$$
f^{n m+k}(x)=\left(f^{m}\right)^{n}\left(f^{k}(x)\right) \rightarrow \xi \quad \text { as } n \rightarrow \infty,
$$

which implies that (ii) holds for $f$. And if $U_{m}$ is an open neighborhood of $\xi$ such that $f^{m m}\left(U_{m}\right) \rightarrow\{\xi\}$, then

$$
U=\bigcap_{k=0}^{m-1} f^{-k}\left(U_{m}\right)
$$

is an open neighborhood of $\xi$ such that, for $0 \leqslant k<m$,

$$
f^{m n+k}(U)=\left(f^{m}\right)^{n}\left(f^{k}(U)\right) \subset f^{n m}\left(U_{m}\right) \rightarrow\{\xi\}
$$

as $n \rightarrow \infty$, implying that (iii) holds for $f$. This completes the derivation of Theorem 2 .

\section{Proof of Theorem 1}

Throughout this section it is assumed that $\lambda \epsilon(0,1)$, that $f: X \rightarrow X$ is a continuous map obeying the conditions (i), (ii), and (iii), and that $\rho_{0}$ is a metric on $X$, giving the correct topology for $X$ and complete if $X$ admits a complete metric. Our aim is to construct a metric $\rho_{\lambda}$, topologically equivalent to $\rho_{0}$ and complete if $\rho_{0}$ is, such that for all $x, y \in X$,

$$
\rho_{\lambda}(f(x), f(y)) \leqslant \lambda \rho_{\lambda}(x, y) .
$$

For the construction, it will be convenient if the neighborhood $U$ of (iii) satisfies

$$
f(\boldsymbol{U}) \subset \boldsymbol{U} .
$$

We show first, therefore, that there exists an open neighborhood $W$ of $\xi$ such that $f(W) \subset W$ and $W \subset U$, the latter implying $f^{n}(W) \rightarrow\{\xi\}$. Then $W$ can replace $U$ in the construction to follow.

Since $f^{n}(U) \rightarrow\{\xi\}$, there is some integer $k$ such that $f^{k}(U) \subset U$. Let

$$
W=\bigcap_{j=0}^{k-1} f^{-j}(U) \subset U .
$$

Then for $x \in W$ we have, for $1 \leqslant j \leqslant k-1, x \in f^{-j}(U)$ and thus $f(x) \epsilon f^{-(j-1)}(U)$; moreover $x \in U$, so that $f^{k}(x) \epsilon f^{k}(U) \subset U$ and thus $f(x) \epsilon f^{-(k-1)}(U)$. Hence $x \in W$ implies $f(x) \epsilon W$, which was to be shown.

We now proceed with the main line of the proof. The construction has three steps. The first step yields a metric $\rho_{M}$, topologically equivalent to $\rho_{0}$ and complete if $\rho_{0}$ is, with respect to which $f$ is nonexpanding in the sense of satisfying the weak version

$$
\rho_{M}(f(x), f(y)) \leqslant \rho_{M}(x, y)
$$

of (3.1). The second step yields a function $d_{\lambda}$ which has all the desired properties except perhaps for satis- 
fying the triangle inequality. This is corrected in the third step, in which $\rho_{\lambda}(x, y)$ is introduced as what

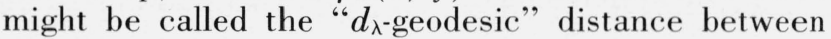
$x$ and $y$.

For the first step, we set

$$
\rho_{M}(x, y)=\max \left\{\rho_{0}\left(f^{n}(x), f^{n}(y)\right): n \geqslant 0\right\} .
$$

That the maximum is finite and actually attained follows from (ii), and (3.3) is obvious. The positive definiteness and symmetry of $\rho_{M}$, as well as $\rho_{M}(x, x)=0$, follow at once from the corresponding properties of $\rho_{0}$. The triangle inequality for $\rho_{M}$ follows from the observation that, for all $n \geqslant 0$,

$$
\begin{aligned}
\rho_{0}\left(f^{n}(x), f^{n}(y)\right) & \leqslant \rho_{0}\left(f^{n}(x), f^{n}(z)\right)+\rho_{0}\left(f^{n}(z), f^{n}(y)\right) \\
& \leqslant \rho_{M}(x, z)+\rho_{M}(z, y) .
\end{aligned}
$$

Thus $\rho_{M}$ is indeed a metric, which must still be shown to be topologically equivalent to $\rho_{0}$, and complete if $\rho_{0}$ is.

From the inequality

$$
\rho_{0} \leqslant \rho_{M}
$$

it follows that any $\rho_{M}$-convergent sequence is also $\rho_{0}$-convergent (with the same limit point). To prove the implication in the opposite direction, note that (iii) implies the existence for each $\delta>0$ of an $N$ such that

$$
\left(\rho_{0} \text {-diam }\right)\left[f^{n}(U)\right]<\delta \quad \text { for } n>N .
$$

For each $x \in X$, it follows from (ii) that

$$
\nu(x)=\min \left\{n \geqslant 0: f^{\prime \prime}(x) \epsilon U\right\}
$$

is finite. Since $f$ is continuous, there is an $\eta>0$ so small that $\rho_{0}(x, y)<\eta$ implies

$$
\begin{aligned}
f^{i(x)}(y) \epsilon U, \rho_{0}\left(f^{j}(x), f^{j}(y)\right) & <\delta \\
& \text { for } 0 \leqslant j \leqslant N+\nu(x) .
\end{aligned}
$$

By (3.2), $f^{n+x^{\prime}+\nu(x)}(x) \epsilon f^{n+.}(U)$ and $f^{n+x^{\prime}+\nu(x)}(y) \epsilon f^{n+x^{\prime}}(U)$ for all $n>0$, so that (3.6) implies

$$
\rho_{0}\left(f^{j}(x), f^{j}(y)\right)<\delta \text { for } j>N+\nu(x) .
$$

Thus $\rho_{0}(x, y)<\eta$ implies $\rho_{M}(x, y)<\delta$. This shows that a sequence which is $\rho_{0}$-convergent to $x$ is also $\rho_{M}$-convergent to $x$, completing the proof of topological equivalence.

Now suppose $\rho_{0}$ is complete. By (3.4), any $\rho_{M^{-}}$ Cauchy sequence is also a $\rho_{0}$-Cauchy sequence, hence is $\rho_{0}$-convergent, and so (by the topological equivalence of the two metrics) is $\rho_{M}$-convergent. Thus the completeness of $\rho_{M}$ follows from that of $\rho_{0}$.

For the second step in the construction, we begin by defining $K_{n}$ to be the closure of $f^{n}(U)$ for $n \geqslant 0$, and $K_{(-n)}=f^{-n}\left(K_{0}\right)$, so that (iii) implies

$$
K_{n} \rightarrow\{\xi\} \quad \text { as } n \rightarrow \infty .
$$

For $x \epsilon K_{0}-\{\xi\}$, set

$$
n(x)=\max \left\{n: x \in K_{n}\right\} \geqslant 0 ;
$$

finiteness is assured by (3.7). Let $n(\xi)=\infty$, and for $x \in X-K_{0}$ set

$$
n(x)=-\min \left\{m: f^{m}(x) \epsilon K_{0}\right\}=\max \left\{n: x \in K_{n}\right\}<0,
$$

which must exist by (ii). Then $d_{\lambda}$ is defined in terms of

$$
c(x, y)=\min \{n(x), n(y)\}
$$

by the formula

$$
d_{\lambda}(x, y)=\lambda^{c(x, y)} \rho_{M}(x, y),
$$

which has the correct limiting form $d_{\lambda}(\xi, \xi)=0$. Then $d_{\lambda}$ satisfies the metric requirements except perhaps for the triangle inequality, and from (3.3) and the fact that $n(f(x)) \geqslant n(x)+1$, we see that $d_{\lambda}$ has the property

$$
d_{\lambda}(f(x), f(y)) \leqslant \lambda d_{\lambda}(x, y)
$$

desired for $\rho_{\lambda}$. We turn now to the third and last step of the construction.

Denote by $\Sigma_{x y}$ the set of chains $\sigma_{x y}=\left[x=x_{0}, x_{1}\right.$, ..., $x_{m}=y$ ] from $x$ to $y$, with associated lengths

$$
L_{\lambda}\left(\sigma_{x y}\right)=\sum_{1}^{m} d_{\lambda}\left(x_{i}, x_{i-1}\right),
$$

and put

$$
\rho_{\lambda}(x, y)=\inf \left\{L_{\lambda}\left(\sigma_{x y}\right): \sigma_{x y} \in \Sigma_{x y}\right\} .
$$

We shall show that $\rho_{\lambda}$ is the desired metric.

That $f$ is a $\rho_{\lambda}$-contraction follows by applying (3.8) to the links $\left[x_{i-1}, x_{i}\right]$ of any chain $\sigma_{x y}$. Clearly $\rho_{\lambda}$ is symmetric and $\rho_{\lambda}(x, x)=0$; the triangle law holds since following a $\sigma_{x y}$ with a $\sigma_{y z}$ yields a $\sigma_{x z}$.

It remains to show that $\rho_{\lambda}$ is positive definite. Consider any $x \neq \xi$ and any $y \neq x$; assume $n(x) \leqslant$ $n(y)$ without loss of generality. If $y \neq \xi$, any chain $\sigma_{x y}$ either lies in $X-K_{n(y)+1}$, or has a last link which leaves $K_{n(y)+1}$ (and possibly is followed by other links), so that

$\rho_{\lambda}(x, y) \geqslant \lambda^{n(y)} \min \left\{\rho_{M}(x, y), \rho_{M}\left(y, K_{n(y)+1}\right)\right\}>0$.

The remaining case, $y=\xi$, is covered by

$$
\rho_{\lambda}(x, \xi) \geqslant \lambda^{n(x)} \rho_{M}\left(x, K_{n(x)+1}\right)>0 ;
$$

thus $\rho_{\lambda}$ is positive definite and indeed a metric, which must still be proved equivalent to $\rho_{M}$.

Let $B_{\nu}=X-f^{-\nu}(U)$ for $\nu \geqslant 0$, so that the definition of $\nu(x)$ implies $\rho_{M}\left(x, B_{\nu(x)}\right)>0$ and $n(x) \geqslant-\nu(x)$. For any $x \neq \xi$, if $y$ obeys

$$
\rho_{M}(x, y)<\delta(x)=\min \left\{\rho_{M}\left(x, K_{n(x)+1}\right), \rho_{M}\left(x, B_{\nu(x)}\right)\right\},
$$


then $n(x) \geqslant-\nu(x)$, so that (3.9) and (3.10) . . the latter with $x$ and $y$ interchanged . . . imply

$$
\lambda^{\prime \prime(x)} \rho_{M}(x, y) \leqslant \rho_{\lambda}(x, y) \leqslant d_{\lambda}(x, y) \leqslant \lambda^{-\nu(x)} \rho_{M}(x, y) .
$$

Choose $k(x)>\max \{0, n(x)\}$ such that $z \in K_{k(x)}$ implies $\rho_{M}(\xi, z)<\rho_{\lambda}(x, \xi) / 2$. Then $\rho_{\lambda}\left(x, K_{k(x)}\right) \geqslant \rho_{\lambda}(x, \xi) / 2$, so that if $y$ obeys

$$
\rho_{\lambda}(x, y)<\rho_{\lambda}(x, \xi) / 2
$$

then only chains disjoint from $K_{h(x)}$ need enter (3.9), implying

$$
\rho_{\lambda}(x, y) \geqslant \lambda^{k(x)} \rho_{M}(x, y) .
$$

In particular, if

$$
\rho_{\lambda}(x, y)<\min \left\{\rho_{\lambda}(x, \xi) / 2, \lambda^{k(x)} \delta(x)\right\}
$$

then with (3.14) and (3.15) this implies (3.12) and hence (3.13) applies. Thus $\rho_{\lambda}\left(x_{n}, x\right) \rightarrow 0$ iff $\rho_{M}\left(x_{n}, x\right) \rightarrow 0$. then

As for $x=\xi$, note first that if $\rho_{M}(\xi, y)<\rho_{M}\left(\xi, B_{0}\right)$,

$$
\rho_{\lambda}(\xi, y) \leqslant d_{\lambda}(\xi, y) \leqslant \rho_{M}(\xi, y) .
$$

Second, for any $\eta>0$, (iii) guarantees an $N(\eta)>0$ such that $\rho_{M}(\xi, z)<\eta / 2$ for all $z \in K_{N(\eta)}$. Then

$$
\rho_{M}(\xi, y)>\eta
$$

implies that $\rho_{M}\left(y, K_{N(\eta)}\right) \geqslant \eta / 2$ and thus that

$$
\rho_{\lambda}(\xi, y) \geqslant \rho_{\lambda}\left(K_{N(\eta)}, y\right) \geqslant \lambda^{N(\eta)} \eta / 2 .
$$

Hence $\rho_{\lambda}\left(\xi, x_{n}\right) \rightarrow 0$ iff $\rho_{M}\left(\xi, x_{n}\right) \rightarrow 0$.
To show that $\rho_{M}$-completeness is preserved, assume that $\left\{x_{n}\right\}$ is a $\rho_{\lambda}$-Cauchy sequence and that $\left(X, \rho_{M}\right)$ is complete. If $\left\{x_{n}\right\}$ does not converge to $\xi$ then since $\rho_{\lambda}$ and $\rho_{M}$ are equivalent, for some $N$ and all sufficiently large $n$,

$$
n\left(x_{n}\right)<N .
$$

Now exactly as above choose $k\left(\left\{x_{n}\right\}\right)=P>\max$ $\{0, N\}$ such that $z \in K_{k(\{x\})}$ implies $\rho_{M}(\xi, z)<\inf$ $\left\{\rho_{\lambda}(x, \xi) / 2, x \in\left\{x_{n}\right\}\right\}=R / 2$. Then since $\left\{x_{n}\right\}$ is a $\rho_{\lambda}$-Cauchy sequence there is an $i>0$ such that

$$
\rho_{\lambda}\left(x_{p}, x_{p+j}\right)<R / 2
$$

for all $p>i$, and using (3.15) with $k(x)=P$, we have

$$
\lambda^{-l} \rho_{\lambda}\left(x_{p}, x_{p+j}\right) \geqslant \rho_{M}\left(x_{p}, x_{p+j}\right)
$$

so that $\left\{x_{n}\right\}$ is a $\rho_{M}$-Cauchy sequence and the proof is complete.

The author thanks A. J. Goldman for his advice and encouragement.

\section{References}

[1] Meyers, P. R., Some Extensions of Banach's Contraction Theorem, J. Res. NBS 69B (Math. and Math. Phys.) No. 3. 179-184 (1965).

[2] Kolmogorov and Fomin, Elements of the Theory of Functions and Functional Analysis 1, 43-45 (Rochester, Graylock, 1957).

[3] Janos, L.. Two operations on distance functions, Notices A.M.S. 11, No. 3, 614-7 (Aug. 1964); A converse of Banach's contraction theorem, Proc. A.M.S. 18, No. 2, 287-289 (1967).

(Paper 71B2 \& 3-202) 